\title{
L'HOMME L'Homme
}

$180 \mid 2006$

Rendre visible

\section{Vous avez dit "modernité" ?}

Modernité, nationalisme et consommation dans un monde globalisé

Denis Monnerie

\section{(2) OpenEdition}

Journals

Édition électronique

URL : http://journals.openedition.org/lhomme/24765

DOI : 10.4000/lhomme.24765

ISSN : 1953-8103

Éditeur

Éditions de l'EHESS

Édition imprimée

Date de publication : 1 décembre 2006

Pagination : 184-196

ISSN : 0439-4216

Référence électronique

Denis Monnerie, « Vous avez dit "modernité" ? », L'Homme [En ligne], 180 | 2006, mis en ligne le 01 janvier 2008, consulté le 01 mai 2019. URL : http://journals.openedition.org/lhomme/24765 ; DOI : 10.4000//homme.24765

(c) École des hautes études en sciences sociales 


\title{
Vous avez dit "modernité"?
}

\section{Modernité, nationalisme et consommation dans un monde globalisé}

\section{Denis Monnerie}

\begin{abstract}
A
VANT MÊME les grandes recherches critiques de Michel Foucault, au cour des meilleurs textes des anthropologues s'ouvre une série d'interrogations systématiques sur des concepts qui sont pourtant nos outils de description et d'analyse. Les réflexions et controverses autour du vocabulaire progressivement mis en place depuis Lewis H. Morgan pour l'étude du domaine de la "parenté » sont l'exemple le plus probant de cette tradition anthropologique de critique et réévaluation. C'est cependant le phénomène inverse - un usage parfois sans distance des découpages linguistiques et culturels occidentaux - qui caractérise une partie de la production anthropologique, particulièrement celle qui vise à la généralisation. Nombre de ces outils de travail et de réflexion sont en effet, dans le contexte même des nations d'origine des anthropologues, des «mots clés " dont Raymond Williams (1976) a bien montré le caractère problématique au niveau sémantique et l'inscription dans des débats de société aux enjeux considérables. Cela les fait parfois ressembler plus à des mots-valises qu'à des concepts aux relations, contenus et/ou contours maîtrisés. Ainsi peut-il apparaître à la fois tout à fait fondé, banal ou carrément faux d'affirmer, de façon très générale, que des sociétés humaines d'ici et d'ailleurs ont en partage nombre d'institutions, de pratiques, de représentations, de dynamiques. En effet, la description même de celles-ci dans le vocabulaire, donc dans les découpages et les axiologies propres à un petit nombre de langues européennes ou occidentales - qui sont parmi les outils fondamentaux de la réflexion en anthropologie sociale et culturelle -, tend à conférer une sorte d'homogénéité à ces institutions, pratiques et représentations dynamiques que nous supposons alors partagées par " nous modernes" et d'autres, alors même que cette notion de modernité, avec d'autres, fait problème. Cela interroge l'anthropologie au moins à deux niveaux, et ses implications épistémologiques ne peuvent être négligées.
\end{abstract}

À propos de Bruce Knauft, ed., Critically Modern: Alternatives, Alterities, Anthropologies, Bloomington-Indianapolis, Indiana University Press, 2002; et de Robert Foster, Materializing the Nation: Commodities, Consumption and Media in Papua New Guinea, Bloomington-Indianapolis, Indiana University Press, 2002. 
Bruce Knauft, dans son introduction à Critically Modern, aborde cette question à propos de concepts classiques de l'anthropologie comme ceux de culture, civilisation, structure et modernité. Ces concepts passent du singulier au pluriel, s'affaiblissent, s'adjectivent. Bruce Knauft souligne aussi que la recherche des délimitations de ces catégories a désormais cédé le pas, que d'autres concepts occupent le devant de la scène: "Le concept de culture est d'abord devenu un réseau de cultures plurielles, puis s'est trouvé mis à l'écart en raison du fait qu'il est lui-même "culturel" [...], [désormais] nous envisageons rarement la "culture" en tant que référent empirique clos avec des frontières définissables" (pp. 35-37). Remarquons en passant que la démarche d'Émile Benveniste où prime l'étude des relations entre ce type de concepts ne semble pas encore avoir franchi l'Atlantique (Benveniste 1969). On notera enfin que certains mots des sociétés étudiées par les anthropologues, et utilisés par eux à des fins de généralisation - totem, tabou, potlatch, mana, etc. -, tendent à présenter un traitement parallèle ${ }^{1}$.

Au nombre de ces notions problématiques, Bruno Latour, dans son essai Nous n'avons jamais été modernes (1997), souligne lui aussi la polysémie et la variété des situations et stratégies d'énonciation du mot «moderne ». Bien d'autres l'ont fait avant lui. Je ne prendrai que deux exemples de cette diversité. Le premier s’insère dans une approche générale critique - féministe - de l'anthropologie, du monde occidental et de la colonisation : Mona Etienne (1980:5) précise que «la dichotomie "traditionnel/moderne" implique un jugement de valeur implicite, et pas seulement en suggérant que "moderne" est en quelque sorte meilleur; cette distinction fait de la société occidentale un idéal et n'est pas très éloignée des idées de "progrès" du XIX siècle». Dans un registre bien différent, et sur un plan heuristique, la démarche de Louis Dumont $(1983,1991)$ se fonde largement sur un contraste moderne/non-moderne qui, dans ses travaux comparatistes sur les diverses formes de «l'individualisme moderne " (France, Allemagne en particulier), prend une forme bien spécifique puisque cette distinction informe l'étude du monde moderne et se révèle, par exemple, particulièrement convaincante dans l'étude du nazisme. Un fait qui, peut-être, en dit long sur les spécificités de notre démarche, est que, dans des domaines bien différents, ces ouvrages sont devenus des classiques, souvent cités et sont à l'origine de développements et de débats contribuant à des avancées ou des rebonds dans notre discipline. Ainsi les usages de "moderne, modernité", posent problème et les deux livres présentés dans cet À Propos affrontent avec lucidité l'après Nous n'avons jamais été modernes, comme on peut dire qu'il y eut un après

1. Sous un autre angle, les travaux monographiques effectués dans la (les) langue(s) locale(s) ou avec une attention soutenue à celle(s)-ci mettent bien souvent en évidence d'une part les inadéquations de nos concepts au niveau de la traduction - ce qui souligne encore le phénomène que je viens de brosser ci-dessus à grands traits -, d'autre part un phénomène en quelque sorte parallèle à celui-ci, à savoir une tendance assez répandue des sociétés et des cultures à articuler, dans leurs propres langues, leurs domaines cruciaux autour de concepts polysémiques. Comme si, de façon assez générale, ici et ailleurs, penser le social, le culturel, le politique, impliquait le recours à des termes polysémiques ou à sémantisme large. 
Le Totémisme aujourd'hui (Lévi-Strauss 1974) ${ }^{2}$. Critically Modern (CM) et Materializing the Nation $(M N)$ - c'est tout leur intérêt - posent un regard critique acéré, soigneusement informé par des ethnographies passionnantes et souvent novatrices sur les notions de "moderne » ou de "modernité », et pour l'un d'eux $(C M)$, le font en partie par rapport aux propositions de Latour ${ }^{3}$. En effet, la perspective de celui-ci peut être comme renversée: si nous n'avons jamais été modernes pouvons-nous repérer des formes spécifiques de modernité autres que celle dont nous nous réclamons? Cela implique une autre forme de retour critique sur la notion de modernité : est-elle, ou non, globale? occidentale ? locale? singulière ou plurielle? Vivons-nous une nouvelle période de "grande transformation" (Polanyi 1983) ? À quelle(s) échelle(s) ? Ces deux livres réagissent à ces interrogations en développant la thèse de ce que l'on pourrait nommer «les modernités multiples » ou « les modernités spécifiques » sur deux fronts : une critique de la notion de modernité et des présentations parfois brillantes qui, à partir d'études ethnographiques, viennent soutenir et approfondir la thèse selon laquelle il existe des modernités multiples et spécifiques.

Critically Modern, ouvrage collectif dirigé par Bruce M. Knauft, est une tentative d'appréciation critique de la notion de modernité se fondant le plus souvent sur des travaux de terrain, mais avec des visées théoriques affirmées. Dans le contexte de la Papouasie-Nouvelle-Guinée, Materializing the Nation de Robert J. Foster travaille la question de la modernité sous trois angles bien définis : la formation de la nation, les phénomènes de consommation et la circulation de l'information moderne, principalement sous l'angle de la publicité.

L'introduction de B. Knauft s'attache d'abord à retracer les publications les plus significatives qui mènent à la vision critique, alternative, de la modernité défendue dans le livre. On peut repérer deux pôles dans ces textes de référence: d'une part, des travaux comme ceux d'Arjun Appadurai, Anthony Giddens, David Harvey (etc.) dont la visée est d'ordre général, et d'autre part, des études se focalisant sur l'étude de "micromodernités». Les modernités critiques, ou alternatives, présentées dans Critically Modern montrent que «les faiblesses de la théorie de la modernité ne doivent pas nous amener à négliger les aperçus pénétrants qu'elle nous procure ». Ainsi des concepts de Giddens : distanciation et désimbrication (disembedding) ${ }^{4}$. Ils sont repris par Foster pour montrer « comment

2. Pour la question du " totémisme ", celle-ci rebondit avec les propositions de Philippe Descola dans Par-delà nature et culture (2005). (Voir également le débat entre Jean-Pierre Digard et Philippe Descola, à propos de l'ouvrage de ce dernier, in L'Homme, 2006, 177-178 : 413-436 [Ndlr].)

3. Un autre aspect de ce débat sur la «modernité » est contextuel dans l'histoire des idées contemporaines. Pendant et après la flambée dite " postmoderne - qui, avec les propositions du philosophe Jean-François Lyotard, a concerné de nombreux domaines intellectuels et artistiques dans les années 1970 à 1990 -, il a été important pour de nombreux anthropologues de se positionner vis-à-vis de ce mouvement et/ou de faire un retour critique sur le «moderne " et la «modernité ».

4. Ce concept est en fait emprunté à Karl Polanyi, le grand absent de ce livre qui n'est cité par aucun des auteurs alors que ses travaux ouvrent la voie à nombre des problématiques développées dans l'ouvrage. 
localement les gens reconfigurent les tendances modernes en fonction de leurs propres objectifs, réalisant ainsi eux-mêmes leurs réimbrications et repositionnements » (CM, pp. 20-21 et 65-72 ; voir aussi $M N$, pp. 131-132; ainsi que Akin \& Robbins 1999 pour la monnaie moderne en Mélanésie).

Le chapitre de Michel-Rolph Trouillot, intitulé "The Otherwise Modern ", présente une réflexion sur les Caraïbes. Il expose bien l'esprit général du volume, qui est celui d'une ambivalence par rapport à la possibilité même d'une approche centrée sur la modernité - concept "universel promu par le monde nordAtlantique, la modernité déguise et déforme tous les "Autres" alors même qu'elle les crée " $(C M$, p. 221). En effet, les universaux promus par ce monde nordAtlantique ont pour caractéristique d'être " toujours prescriptifs ", ce qui découle du fait qu'il s'agit de «la projection d'une expérience historique limitée - celle du monde nord-Atlantique - sur la scène mondiale». Cette vision, qui rejoint celle développée deux décennies auparavant par Mona Etienne (voir supra), est appliquée à l'expérience historique de l'esclavage aux Caraïbes, monde qui fut «moderne dès le premier jour» (p. 232), car les spécialisations professionnelles des esclaves et surtout l'exploitation de leurs jardins vivriers y ont produit des "identités modernes» (p. 229). En effet, la vente des produits de ces jardins possédés individuellement transforme les esclaves en consommateurs, et cela est vrai surtout des femmes qui contrôlent cette distribution sur les marchés. Le soin qu'elles apportent à leurs dispendieux vêtements, leurs nombreux signes de coquetterie ont frappé tous les observateurs : "Si la modernité est aussi la production de soi individuels à travers des structures de production et de consommation, les esclaves des Caraïbes étaient modernes, car ils avaient intériorisé des idéaux d'amélioration personnelle à travers le travail, la possession de marchandises spécifiques et l'identification à celles-ci » (p. 230).

Pour M.-R. Trouillot, les objections qui peuvent être faites à cette analyse ne font que renforcer son scepticisme envers les théories de la modernité. Finalement, ce qui doit être analysé plus précisément et surtout différemment c'est « la relation entre la géographie de la gestion et celle de l'imagination qui ensemble ont insufflé et sous-tendu le développement du capitalisme mondial ». Ce que nous enseigne le monde caraïbe c'est «que la modernité n'a jamais été - n’a jamais pu être - ce qu'elle prétend être» (p. 234).

Dans «Braquer les bus et récolter des biscuits au fromage », Holly Wardlow nous transporte chez les Huli de Tari, en Papouasie-Nouvelle-Guinée. L'originalité de son analyse est de mettre en relation certaines pratiques féminines avec celle de ces braqueurs - des jeunes gens qui s'attaquent aussi aux marcheurs, aux boutiques et même aux passagers des avions - que l'on connaît là-bas sous le nom de rascals. Elle montre comment, avec toutes leurs différences, le vol et le fait de "planter» des nourritures industrielles sont deux formes sexuées (gendered) de pratiques issues de la modernité, toutes deux en relation avec le travail. Elles résultent de la "dévaluation d'un travail physique et socialement intégré, dans le 
contexte de relations sociales qui prennent des formes de plus en plus marchandes " (p. 145). Si les pratiques violentes et très répandues des rascals sont désormais bien décrites, celles de certaines femmes huli sont plus spécifiques. L'auteur prend l'exemple d'une femme qui, au jardin, enterre des biscuits au fromage pour que sa fille les déterre pendant qu'elle-même récolte des patates douces. Il ne s'agit pas là d'une forme de rapport aux marchandises qui privilégie des pratiques magiques ou occultes. Bien au contraire, pour cette femme, l'enseignement que doivent en retirer les filles est que l'activité productive est essentielle et que nourritures et biens modernes n'apparaissent pas comme par enchantement. Alors "que la relation entre le travail et la richesse a été effacée, cette femme essaie de la réintroduire en réinsérant le travail productif dans l'équation, forçant une marchandise produite industriellement à prendre la forme d'un produit horticole qui exige d'être récolté» (p. 157). Par contraste, pour les rascals, "la relation entre la richesse et le travail physique, socialement intégré, n'est plus tenable, mais [...] simultanément, le travail salarié ne peut pas le remplacer. Ces deux pratiques - enterrer de la nourriture achetée et considérer le vol comme une sorte de salaire - essaient de rendre à nouveau "transparente" la relation entre la production et la richesse, mais ce sont les femmes qui s'en tiennent à la valeur innée et à la puissance du travail physique de [l'horticulture] de subsistance, alors qu' ironiquement, pour les hommes, puisque le travail socialement intégré n'est plus indispensable à l'acquisition des richesses ou à la production de significations ou de valeur sociales, n'importe quelle activité peut potentiellement être considérée comme un travail pour autant qu'elle procure de l'argent" (pp. 160-161). C'est ainsi que les femmes et les hommes huli se situent différemment par rapport à la même «modernité locale» (p. 163).

Debra Spitulnik nous propose des « réflexions sur la place des témoignages linguistiques en ethnographie ». Le terrain est la Zambie, l'ethnographie concerne d'une part des émissions de radio et d'autre part les changements de la langue bemba dans un contexte multilingue. Deux types de faits linguistiques sont visés par l'auteur : ceux, dénotatifs, qui réferent à une conception locale de la modernité, et ceux, pragmatiques, qui ont pour fonction de signaler que le locuteur revendique une identité sociale moderne. Il s'agit, comme chez la plupart des auteurs de ce volume, «de prendre la modernité comme un problème ethnographique et non comme une catégorie analytique englobante» (p. 200). Les analyses sont précises, mais sans jargon pouvant rebuter un non-linguiste. Parmi les éclairages qu'apporte D. Spitulnik figure l'étude d'un verbe, ukuca (-ca), dont la seule signification ancienne référait au lever du soleil (to dawn), et qui, avec la colonisation, est devenu polysémique pour en venir à signifier "être éclairé, éveillé, alerte, à la mode ", etc. Le discours colonial de la Rhodésie est à l'origine de ce phénomène, les "Africains étaient considérés comme étant "dans le noir" et "assoupis" ", et les Européens sont venus leur apporter "des technologies, pratiques et croyances destinées à "les réveiller" et les "éclairer" ". Le mot bemba, qui est utilisé pour traduire ces notions coloniales, était précisément $u k u c a$ : «Ce 
qui fut autrefois une façon de décrire la modernité du point de vue européen colonial dominant est maintenant l'une des façons les plus répandues de décrire la modernité parmi les Zambiens eux-mêmes " (p. 209). Cette remarquable étude du double codage linguistique de la modernité et de l'intégration des catégories coloniales est une belle défense et illustration de l'approche ethnolinguistique appliquée à la compréhension de cette forme de la modernité.

Donald Donham, dans son chapitre « $\hat{E}$ tre moderne dans un monde capitaliste ", nous invite, lui, à prendre nos distances vis-à-vis de la "prolifération du concept de modernité et en particulier de la tendance à le pluraliser... ", une tendance conforme à la "structure et aux sentiments dominants en anthropologie " - le relativisme - qui suggère que chaque modernité est sur un pied d'égalité avec les autres, mais laisse de côté « d'énormes différences de pouvoir et de richesse » dans le système global (p. 242). Car la modernité est aussi partie prenante de «sphères publiques locales dans lesquelles certains acteurs [...] invoquent des aspects de la modernité dans leur tentative d'accession au pouvoir» (p. 244). Le terrain est l'Éthiopie, et l'approche consiste en un retour critique de D. Donham sur le livre qu'il a publié en 1999, Marxist Modern: An Ethnographic History of the Ethiopian Revolution. Cette démarche est centrée sur une analyse de la christianisation menée depuis les années 1930 par une secte canadienne fondamentaliste (The Sudan Interior Mission, SIM), se caractérisant à l'origine par des positions antimodernes. Cette secte s'est bien implantée dans le sud du pays. Toutefois ses adeptes, qui pour avoir accès à la Bible avaient appris à lire la langue nationale (l'amharique), se considéraient, eux, comme des " modernistes locaux " et décidèrent d'opérer deux ruptures: l'une, très radicale avec la tradition; l'autre, contre les élites du Nord très généreusement soutenues par une aide des États-Unis qui, dans les années 1950 et 1960, donnèrent à l'Éthiopie autant qu’à tous les autres pays africains pris ensemble. Cela rend compte de deux faits qui peuvent sembler contradictoires : les paysans révolutionnaires du Sud venaient presque tous d'un milieu chrétien évangélique mais, dans le contexte de la bipolarisation mondiale contre les États-Unis, adoptèrent des positions issues du marxisme. Les " copies et anticopies» de la modernité ne deviennent "claires que si de telles idées sont placées dans le contexte du capitalisme global, avec ses différences de pouvoir, de richesse et d'espérance de vie » (p. 255).

Après seize années d'absence, Bruce Knauft, de retour sur son terrain chez les Gebusi de Papouasie-Nouvelle-Guinée, est confronté à deux phénomènes bien connus des anthropologues et mis ici en résonance: "la réinvention de la tradition [qui] est en fonction directe du processus complémentaire qui consiste à devenir localement moderne» (p. 106) est indissociable du fait que les Gebusi ont des rapports à la modernité de plus en plus partagés, que «simultanément ceci produit une panoplie de réactions spécifiques au niveau culturel» (p. 134), et qu'en même temps "ils sont de plus en plus divers culturellement, de façon typique, plus inégaux qu'ils n'étaient autrefois» (p. 106). 
Cinq autres chapitres de Critically Modern présentent des études, dont certaines sont d'un grand intérêt. Robert Foster, par exemple, s'intéresse aux «marchandages avec la modernité " en Papouasie-Nouvelle-Guinée et ailleurs. Il reprend des propositions de Anthony Giddens pour les assouplir, en effet «si nous pouvons montrer l'aspect multiple de la modernité, pourquoi ne pourrionsnous pas pluraliser Giddens?» (p. 65). Son étude est ici centrée sur les usages de la monnaie papoue moderne, un thème qui, dans ce pays, a retenu de nombreux anthropologues et est devenu emblématique des formes multiples et localisées de la modernité. Ivan Karp, dans "Développement et personne", nous emmène brièvement sur un terrain kenyan et, à cette occasion, nous fait entendre la voix de Jomo Kenyatta, mais aussi celles de Carlos Fuentès et de J. M. Coetzee. Pour I. Karp, les discours sur le développement et la personne impliquent que cette dernière soit définie au moyen de stéréotypes qui définissent des humains - individus ou groupes - susceptibles d'être « développés ». De cette façon, après avoir donné forme, sa forme, à ce matériau humain, qui bien sûr doit être transformé, le discours du développement va pouvoir définir les processus nécessaires à cette transformation. Dans un chapitre consacré à la "modernité et autres traditions ", Jonathan Friedman revient sur plusieurs de ses publications, critique les positions des Comaroff (1999) et propose deux « catégorisations » de la modernité. D’une part « la modernité contemporaine [...], situation d'intégration dans l'économie capitaliste mondiale », et d'autre part "la modernité dans un sens structurel [qui elle] fait référence aux paramètres culturels de l'espace d'expérience capitaliste, une excroissance de la marchandisation des relations sociales» (p. 307). Le texte de Lisa Rofel intitulé "Les fantasmes masculins de la modernité » est principalement consacré à une lecture critique du livre de Michael Hardt \& Toni Negri, Empire 5 . Pour elle, "la politique sexuelle de Empire qui se coule dans une structure narrative en forme d'allégorie biblique nous pousse de façon irrésistible à nous tourner vers d'autres productions culturelles populaires» (p. 188) avec lesquelles ce livre partage de nombreux traits communs, à savoir les films de la série Star Wars de George Lucas.

C'est donc vraiment une vision critique, kaléidoscopique, de la modernité - de modernités plurielles et plus ou moins localisées - qui nous est proposée dans ce très intéressant ouvrage où étudiants avancés et chercheurs trouveront de précieuses informations, de nombreuses suggestions de recherche; on y trouvera aussi des bibliographies où apparaissent beaucoup de noms et de travaux importants presque jamais cités dans les publications francophones sur cette question. Mais, sur ce genre de problème comme sur d'autres, le point de vue français, on le sait, tend le plus souvent à privilégier le "politique " alors que les anthropologues qui ont contribué à cet ouvrage regardent, eux, plutôt du côté de la "culture ». En plus des nombreuses propositions théoriques contenues dans ces textes, je suis tenté par une suggestion supplémentaire qui concerne le discours scientifique. 
Dans ce livre, comme ailleurs, l'idée de modernité se trouve comme éclatée, alors même que la pertinence et l'aspect convaincant de la plupart des analyses n'en sont pas pour autant amoindris. Il ne s'agit là pas seulement de l'intérêt d'une approche critique qui envisage la thématique de la modernité comme une véritable interrogation. Au-delà, $j$ 'avancerai que ce livre renforce l'idée que la pertinence et la rigueur des propositions scientifiques de l'anthropologie sociale et culturelle ne se mesurent sans doute pas à l'aune de la définition très stricte de concepts isolés - «modernité » est clairement un mot polysémique - ou de propositions relativement brèves, très épurées, à la façon des théorèmes, mais à celle de textes plus longs laissant une respiration au développement des argumentations et éventuellement à la (re)configuration des notions; les indispensables critères de pertinence et de rigueur s'appliquant alors à ces segments de discours relativement longs. Comme d'autres enjeux des sciences sociales, la «modernité» tend à n'être qu'une sorte de pseudo-concept qui, s'il est pris isolément et considéré a priori comme rigoureux, tend à fonctionner au mieux comme une boîte noire, au pire comme un thème de méditation assez flou. Critically Modern, surtout dans ses meilleures contributions, réussit à replacer la modernité dans son champ de pertinence : celle-ci est de l'ordre du questionnement, et ce livre en débusque certains aspects occidentalocentrés et les valeurs implicites qui y sont associées. Il lui fait subir une salutaire torsion, un décapage et des enrichissements bienvenus ; que les réponses à ces questionnements renouvelés nous étonnent parfois est une marque de la qualité de cette démarche critique.

Ce type d'approche me semble aussi attirer l'attention sur un fait crucial : il est pour le moins imprudent ou prématuré de désigner une caractéristique unique à la modernité ou à la tradition, ou à la coutume, pour l'ensemble de l'Océanie, ou de la Mélanésie - et vraisemblablement pour d'autres régions que je connais moins. Paraphrasant Jean Baudrillard, on pourrait avancer que modernité, tradition ou coutume ne sont pas des concepts d'analyse - caractérisés par des lois mais des notions autour desquelles nous rassemblons, principalement du point de vue des anthropologues, des Européens de «l'Atlantique Nord » comme le dit Trouillot, des traits significatifs des sociétés qui nous intéressent. À cet égard, il me semble significatif qu'en Polynésie ou en Mélanésie, les notions de tradition et de coutume (ainsi que leurs dérivés) prennent des sens bien différents exprimant souvent les points de vue contrastés des autochtones d'une part et des "expatriés», parfois de certains anthropologues, de l'autre. De sorte que les visions émiques et étiques de ces notions ne sont pas nécessairement en adéquation. Les nombreuses études de qualité dont nous disposons désormais sur la Papouasie-Nouvelle-Guinée contemporaine illustrent ce phénomène. Car le processus d'édification de cette jeune nation moderne est contemporain du «boom anthropologique » qui a marqué ce pays fascinant. Ainsi disposons-nous à ce sujet d'ethnographies très riches, diverses à la fois par les régions étudiées et les perspectives de leurs auteurs. 
C'est précisément la Papouasie-Nouvelle-Guinée et plus précisément encore les spécificités du processus de construction de cette jeune nation - indépendante depuis 1975 sous la férule du journaliste politicien Michael Somare et d'une Australie pressée de décoloniser - qui sont au centre des analyses de Robert Foster dans Materializing the Nation. Robert Foster est sans doute l'un des meilleurs de ceux qui ont à la fois pratiqué l'étude ethnographique de la modernité en Océanie et observé ses développements et incertitudes théoriques avec un vrai recul (voir sa contribution dans CM, et surtout Foster 1991). Sous sa plume, l'étude de la question de la construction de la nation revêt un grand intérêt.

La Papouasie-Nouvelle-Guinée est une nation généralement considérée comme fragile, ce que montre, outre le phénomène assez généralisé des rascals évoqués ci-dessus, la très longue guerre menée par les habitants de la région de Panguna, dans la grande île de Bougainville, contre les compagnies minières qui ont ravagé leur environnement et contre le pouvoir central qui entendait engranger l'essentiel des profits de cette exploitation. Alors que nombre des travaux de sciences sociales des années 1950 et 1960 sur la question des jeunes nations nous apparaissent aujourd'hui comme de véritables pamphlets en faveur des indépendances des pays colonisés ${ }^{6}$, des travaux comme ceux de R. Foster bénéficient aujourd'hui d'un certain recul, tout en demeurant dans le contemporain, donc traitant de ce qui est immédiatement observable au niveau du vécu, avec les outils de l'ethnographie. Materializing the Nation ne souffre pas d'être composé de chapitres déjà publiés, tant le dessein de l'auteur est bien affirmé. Il s’agit de répondre à la "vieille question de Renan, "Qu'est-ce qu'une nation ?" ", mais surtout de reprendre cette question dans le cadre de la globalisation (p. 1). Pour la Papouasie-Nouvelle-Guinée, l'exemple des lectures, différentes localement, du drapeau national ou de l'aspect iconique de la monnaie est désormais bien connu. L'intérêt de la démarche de R. Foster est qu'elle emprunte des chemins assez radicalement différents de ceux de la politologie ou de l'ethnologie classiques - en particulier des thématiques uniquement centrées sur les rapports à l'État - pour se concentrer sur les usages et représentations sociales des marchandises et de la publicité. "Ce livre», annonce l'auteur, "montre comment "la nation" se matérialise sous la forme d'images médiatiques et de biens de consommation et de quelle façon la culture nationale de Papouasie-NouvelleGuinée prend forme - mais le fait-elle vraiment ? - conjointement avec la diffusion de nouvelles technologies dans les médias et de nouveaux domaines pour la consommation des marchandises. Ce faisant, ce livre nous parle de ce qu'est au quotidien la construction de la nation, aujourd'hui et dans le passé, en PapouasieNouvelle-Guinée et ailleurs » (p. 2). La spécificité de la Papouasie-Nouvelle-Guinée comme "État faible» est soulignée. Ce qui la caractérise et fait l'originalité de ce livre, c'est que, selon R. Foster (suivant Kelly 1995), l'État n'est pas le seul

6. Les remarquables textes de Frantz Fanon me semblent emblématiques de cette tendance poussée à l'extrême. Le désormais célèbre livre de René Dumont L'Afrique noire est mal partie (1962) ne déroge pas à cette règle, mais, à cette époque, il se distingue par sa (rare) exigence de lucidité face à l'avenir. 
critère de définition et surtout d'existence d'une nation, d'un sentiment national. Cela ouvre à des visions ethnographiquement diverses de "la nation" - ce que suggèrent aussi des exemples comme ceux de l'Italie ou de l'Indonésie (p. 110). Mais au-delà du cas de cette jeune nation se caractérisant par sa faiblesse étatique, $\mathrm{R}$. Foster signale que « les politiques fiscales des agences internationales de régulation telles que la Banque Mondiale et le FMI provoquent une angoisse largement répandue, de Seattle à Prague, aussi bien que dans les rues de Port Moresby, la capitale de la Papouasie Nouvelle Guinée » (p. 4). La part d'ironie comparatiste de ce livre ne doit pas être sous-estimée.

L'ouvrage est organisé en trois parties. La première est assez classiquement consacrée à des projets nationaux promus par l'État, concernant la justice, la criminalité et la monnaie. La deuxième s'intéresse à la part de la publicité dans la construction du sentiment national, aux niveaux personnel et collectif, montrant le rôle des marchandises diffusées par le capitalisme consumériste globalisé et leurs connivences avec l'individualisme, la valorisation du corps, la santé. Cette partie du livre est centrale dans tous les sens du terme. La troisième partie élargit la perspective en étudiant les interactions existant entre la construction de la nation et la globalisation contemporaine, en soulignant la façon dont les «marchandises globales deviennent le matériau de construction de la nation et comment les significations de ces marchandises sont objets de négociation aussi bien de la part des spécialistes du marketing que de celle des consommateurs" (p. 21). L'intérêt porté aux biens de consommation, à leurs aspects concrets et à leurs interprétations est mis en valeur par de nombreuses photographies et reproductions, et certaines affiches vantant des boissons gazeuses sont de véritables pièces d'anthologie. L'attention portée aux paroles des chansons pop - démarche de moins en moins rare chez les anthropologues - doit aussi être mentionnée : le texte enjoué de There Goes My Pay, succès de Louie Warupi, donne la note finale au chapitre sur les médias commerciaux.

Ironiquement, le chapitre consacré à la construction commerciale des « nouvelles nations " s'ouvre sur des réflexions portant sur les États-Unis. R. Foster analyse un dessin satirique du New Yorker, des représentations concernant un modèle de chaussures Reebok - carrément qualifiées par les vendeurs et les consommateurs «d'hymne national » - et, surtout, affirme que, dans ce pays, la translation « du rituel politique au rituel commercial [...], témoigne d'une éclipse de l'État au profit du marché comme référence principale de l'appartenance nationale» (p. 109). On y trouve aussi une référence aux processus d'américanisation des immigrants juifs d'Europe de l'Est par la marchandise (pp. 122-123) et l'exemple de cet aviateur américain de la Seconde Guerre mondiale qui, dans ses mémoires, déclare que la motivation principale de son premier combat victorieux était «l'Amérique, la Démocratie [et un] Coca-Cola» (p. 118), témoignage qui évoque celui de ces soldats américains au Vietnam, isolés dans la forêt sous le feu de l'armée vietcong, se battant entre eux pour les rations comprenant les meilleurs desserts ${ }^{7}$. Puis nous

7. L'exemple n'est pas dans le livre de Foster, mais m’a été raconté par un photographe d'une agence américaine. 
nous éloignons des États-Unis, avec un « voyage imaginaire » qui nous mène du Sri Lanka et ses loteries à l'Espagne avec l'exposition universelle de Séville en 1992, en passant par les îles Cook et son tourisme ethnique. Pour R. Foster, les nations sont aussi des communautés de consommation. En Papouasie-Nouvelle-Guinée, alors que l'État peine à s'imposer, des produits de consommation de masse se sont répandus dans tout le pays fournissant aux «publicitaires des supports qui leur permettent d'imaginer la nation comme étant le marché auquel ils aspirent " (p. 119). Ce processus en boucle où la publicité imagine la nation et la nation se définit par rapport aux marchandises ainsi promues sous-tend la thèse de l'auteur selon laquelle des interactions très fortes entre produits de consommation, marché et nation définissent une sorte de strate constitutive de l'identité et des pratiques nationales qui existe partout - et en premier lieu aux États-Unis - mais, en raison de la faiblesse de l'État, se trouve placée au centre de la construction de la nation en PapouasieNouvelle-Guinée. Pour ce pays, la légendaire rivalité entre Pepsi-Cola et Coca-Cola fournit à ce chapitre l'essentiel de ses analyses. Par contraste avec Nicholas Thomas, qui pense que ce type de rapport à la marchandise révèle un «échec du projet moderniste dans le Pacifique Sud» (p. 124), R. Foster avance l'idée qu'il s'agit, dans ce cas précis, d'une pondération différente de ces diverses strates de la modernité qui, ensemble, caractérisent la construction de la nation : outre les classiques rapports au territoire, à l'État, au sentiment patriotique (etc.), les pratiques de consommation sont partie intégrante d'une "forme faible de la communauté [...] offerte par le marché global» (p. 126). Cependant, dans le cas qui nous occupe, celui de la consommation de boissons, ou de nourritures, nous rejoignons une forme plus forte, très répandue et ancienne de l'affirmation alimentaire des groupes sociaux, un point qui, il me semble, n'est pas assez souligné par R. Foster. La raison en est certainement que son principal souci dans ce chapitre est de signaler que ces phénomènes méritent "notre attention la plus soutenue avant que nous décidions que les perspectives des "nouvelles nations" relèvent de l'impasse" (p. 127).

Serions-nous alors dans la configuration décrite par Epeli Hau'ofa selon qui les habitants du Pacifique «ne peuvent faire comme si le système global n'existait pas, mais [...] peuvent contrôler certains aspects de ses interférences néfastes et saisir toutes les opportunités qui se présentent pour créer [leurs] propres espaces" (p. 131) ? Pour R. Foster, c'est là un exemple d'un vieux problème anthropologique, celui de la "structure et de l'activité des agents sociaux (structure and agency), de la façon dont on rend compte des actions des humains dans des conditions dont ils ne sont pas les créateurs directs ». Comme l'ont aussi montré Hau' ofa (1994) et Sahlins (1993, 1999), la globalisation implique pour les habitants du Pacifique Sud « une expansion de leur monde de référence, [mais] n’implique pas nécessairement que celui-ci soit englobé par un autre monde » (p. 133). Certains traits anciens des sociétés d'Océanie ont préparé la voie, comme le circuit d'échanges de la kula, dans le Massim, en Papouasie-Nouvelle-Guinée, pour lequel Nancy Munn a montré comment la renommée personnelle des participants dépend de relations, médiatisées par des objets, avec des partenaires vivant sur des îles lointaines (1990). Dans une perspective comparable, à l'extrême nord de la Nouvelle-Calédonie, les 
cérémonies de relations dans le vieux réseau régional Hoot ma Whaap peuvent être transformées pour être utilisées dans le cadre de la lutte anticoloniale ou dans celui des relations avec l'Église catholique, ce qui a pour visée d'imposer aux représentants de l'État ou de l'Église de s'exprimer dans un cadre cérémoniel qui met en relief l'autochtonie du peuple kanak (Monnerie 2005). C'est ainsi que chez les Urapmin, un petit peuple qui se considère comme ayant été abandonné par l'État et les Églises, l'influence, considérable, des idées chrétiennes millénaristes montre que l'intégration d'éléments externes venus d'au-delà de la Papouasie-NouvelleGuinée, contribue à transcender les limites de leur propre infériorité supposée par l'affirmation d'une appartenance transnationale. Mais le prix à payer pour cette relation directe et prestigieuse avec la religion et le Dieu blanc est une conscience aiguë de leur «échec» à éviter le péché et à se plier à la volonté de ce Dieu (pp. 136-137, d'après une étude de Joel Robbins 1998). Remarquons toutefois que ces relations ambiguës à l'ailleurs de la religion chrétienne millénariste sont, en définitive, vecteurs de dévalorisation pour les Urapmin. Elles se distinguent donc radicalement, pour les significations et l'orientation des valeurs, de ces ailleurs que sont les îles lointaines, si cruciales pour l'acquisition de la renommée à long terme des participants à la kula. L'ouvrage s'achève sur quelques propositions visant à définir les nouvelles formes de la pratique du terrain ethnographique répondant aux conditions contemporaines d'observation de ce type de phénomènes sociaux où le local se construit et se définit en partie au moins à travers le global.

Comme l'a montré Jean-Pierre Warnier (2004, 2005), et contrairement à des craintes et des clichés assez répandus, la globalisation n'entraîne pas nécessairement une homogénéisation des actes et représentations sociales dans tous les domaines. Les études ethnographiques récentes attestent que les pratiques et réinterprétations de nombreux phénomènes d'emprise mondiale - comme nous venons de voir pour la consommation des boissons gazeuses - se développent localement de façons divergentes pour produire de la diversité. Je crois qu'une question fondamentale est de savoir s'il s'agit d'un phénomène à moyen terme, transitoire, qui, après avoir permis dans un premier temps de sécuriser les importations, les implantations et les flux mondialisés s'estompera pour céder à la poigne de fer du capitalisme et d'une idéologie de la marchandise s'affirmant en définitive comme globale. Je risquerai un parallèle avec les pénétrations coloniales. Elles se sont le plus souvent caractérisées par leur souplesse et leur diversité dans les premières années pour ensuite s'intensifier, se figer, et s'acharner à transformer parfois de façon très profonde les peuples ainsi soumis - bien souvent aussi, ne l'oublions pas, pour les exterminer directement ou indirectement.

Sommes-nous donc dans une telle perspective transitoire dont nous ne pouvons pas prédire les développements ultérieurs? Ou bien les phénomènes décrits dans ces deux livres sont-ils au contraire de nouveaux exemples de la résilience des groupes humains? Puisque ces auteurs se situent sur le plan de la "culture", 
l'exemple de la disparition des très nombreuses langues locales n'incite pas à l'optimisme, plutôt à une grande prudence. Les observations ethnographiques actuelles et futures permettront peut-être de déterminer les tendances de l'avenir - tendances qui ne manqueront pas d'être en dernière instance plus ou moins largement infléchies par les résistances et les oppositions qui, dans le monde entier, visent implicitement ou explicitement à refuser la forme actuellement la plus puissante de globalisation et à en proposer d'autres qui se veulent différentes (Edelman 2001 : 304-309). Là aussi, solidement appuyée sur des ethnographies pratiquées à des échelles différentes (Foster in $C M: 75$ ), l'anthropologie sociale et culturelle devrait avoir un rôle essentiel à jouer dans la compréhension fine et réaliste de ces phénomènes et de leurs trajectoires.

\author{
Département d'ethnologie \\ Université Marc-Bloch, Strasbourg \\ monnerie@umb.u-strabg.fr
}

MOTS CLÉS/KEYWORDS: modernitélmodernity - globalisation/globalization - consommation/consumption - médias/media - construction nationale/national construction - concepts anthropologiques/anthropological concepts - Papouasie-Nouvelle-Guinée/Papua New Guinea.

\title{
BIBLIOGRAPHIE
}

Akin, David \& Joel Robbins, eds

1999 Money and Modernity: State and

Local Currencies in Melanesia. Pittsburgh, University of Pittsburgh Press.

Benveniste, Émile

1969 Vocabulaire des institutions indoeuropéennes, 1 et 2. Paris, Minuit.

Comaroff, Jean \& John

1999 «Occult Economies and the Violence of Abstraction : Notes from the South African Postcolony ", American Ethnologist $26: 279-303$.

Descola, Philippe

2005 Par delà nature et culture. Paris

Gallimard.

Donham, Donald

1999 Marxist Modern: An Ethnographic

History of the Ethiopian Revolution. Berkeley, University of California Press.
Dumont, Louis

1983 Essais sur l'individualisme: une perspective anthropologique sur l'idéologie moderne. Paris, Le Seuil.

1991 Homo aequalis, 2 : L'Idéologie

allemande: France-Allemagne et retour. Paris,

Gallimard.

Dumont, René

1962 L'Afrique noire est mal partie. Paris, Le Seuil.

Edelman, Marc

2001 «Social Movements: Changing

Paradigms and Forms of Politics ", Annual

Review of Anthropology 30 : 285-317.

Etienne, Mona \& Eleanor Leacock, eds

1980 Women and Colonization:

Anthropological perspectives. New York,

Praeger. 


\section{Foster, Robert J.}

1991 "Making National Cultures in the

Anthropology $20: 235-260$.

Hau'ofa, Epeli

1994 "Our Sea of Islands ", Contemporary Pacific 6 (1) : 148-161.

1998 "The Ocean in Us", Contemporary

Pacific 10 (2) : 392-410.

Kelly, John

1995 "The Privileges of Citizenship :

Nations, States, Markets and Narratives ", in Robert J. Foster, ed., Nation Making :

Emergent Identities in Postcolonial Melanesia.

Ann Arbor, University of Michigan Press :

253-273.

Latour, Bruno

1997 Nous n'avons jamais été modernes:

essai d'anthropologie symétrique. Paris,

La Découverte.

Lévi-Strauss, Claude

1974 Le Totémisme aujourd'hui. Paris,

Presses universitaires de France.

Monnerie, Denis

2005 La Parole de notre Maison: discours et cérémonies kanak aujourd'hui, NouvelleCalédonie. Paris, CNRS Éd. - Éd. de la

Maison des sciences de l'homme.

\section{Munn, Nancy}

1990 "Constructing Regional Worlds in

Experience: Kula Exchange, Witchcraft and Gawan Local Events ", Man n. s. 25 : 1-17.

\section{Polanyi, Karl}

1983 La Grande Transformation : aux origines politiques et économiques de notre temps. Paris, Gallimard.

Robbins, Joel

1998 "On reading "World News" :

Apocalyptic Narrative, Negative

Nationalism and Transnational Christianity in a Papua New Guinea Society ", Social Analysis 42 (2) : 103-130.

Sahlins, Marshall

1993 «Goodbye to Tristes Tropes:

Ethnography in the Context of Modern

World History ", Journal of Modern History

$65: 1-25$.

1999 "What is Anthropological

Enlightenment? Some Lessons of the

Twentieth Century ", Annual Review of

Anthropology 28 : I-XXIII.

\section{Warnier, Jean Pierre}

2004 [1999] La Mondialisation de la culture. Paris, La Découverte.

2005 «Mondialisation, disjonctions, émiettements culturels, terrain : les enjeux du militantisme ethnologique ", Parcours anthropologiques 5 : 5-9.

\section{Williams, Raymond}

1976 Keywords: A Vocabulary of Culture and Society. London, Fontana. 